CERN-TH.6777/93 POLFIS-TH.24/93

\title{
STRING QUANTUM SYMMETRIES FROM PICARD FUCHS EQUATIONS AND THEIR MONODROMY *
}

\author{
R. D'AURIA \\ Department of Physics, Politecnico di Torino \\ and INFN Sezione di Torino, Torino, Italy \\ S. FERRARA \\ CERN, 1211 Geneve 23, Switzerland
}

\begin{abstract}
**
Local and global properties of the moduli space of Calabi-Yau type compactifications determine the low energy parameters of the string effective action. We show that the moduli space geometry is entirely encoded in the Picard-Fuchs equations for the periods of the CalabiYau $H^{(3)}$-cohomology.
\end{abstract}

* Supported in part by DOE grants DE-AC0381-ER500050, DOE-AT03-88ER40384, task E and by MURST. 


\section{Introduction}

It is well known that among the possible vacua of heterotic string theory the $(2,2)$, $\mathrm{c}=9$ world-sheet superconformal field theories (SCFT) are of prominent importance since they give rise to $\mathrm{N}=1$ supersymmetry in four dimensions, which is currently believed to be a necessary ingredient of the low-energy effective Lagrangian 1 .

In general it is quite difficult to compute the correlators of a Superconformal Field Theory (SCFT) and usually more insight into their structure can be gained by using the Landau-Ginzburg approach to $\mathrm{N}=2 \mathrm{SCFT}^{2}$ or else in the context of the $\mathrm{N}=2$ topological field theory ${ }^{3}$. The description of the $(2,2)$-string vacua in terms of the Landau-Ginzburg superpotential (possibly in its twisted topological version) is strictly related to the geometrical description in terms of the compactification of the heterotic string on a Calaby-Yau 3-fold (C.Y.) and in fact the determination of the physical low-energy parameters in both frameworks can be performed by using the same techniques of algebraic geometry ${ }^{4,5}$. One is actually faced with the problem of computing such low-energy quantities in terms of the properties of the moduli space of a given SCFT. Moduli space is the space of all the marginal deformations of the underlying $\mathrm{N}=2$ SCFT or, in the geometrical picture of Calabi-Yau compactifications, is the space of the parameters $\varphi^{\alpha}, \psi^{a}$ describing respectively the deformations of the Kähler class and/or of the complex structure of the C.Y. manifold ${ }^{6}$. In the low energy theory the moduli parameters $\varphi^{\alpha}(x), \psi^{a}(x)$ appear as neutral massless scalar fields with vanishing potential.

The low energy objects that we are interested in as functions of the moduli are: i) the moduli and family metrics; ii) the Yukawa couplings. Another important moduli dependent effect is: iii) the running of the gauge couplings due to one-loop stringy effects.

These quantities appear in the effective $N=1$ Lagrangian in the following form:

i)

$$
g_{i \bar{j}} \partial_{\mu} M^{i} \partial_{\nu} \bar{M}^{\bar{j}} G^{\mu \nu}
$$




$$
\begin{aligned}
& g_{\alpha \bar{\beta}}^{(27)} \partial_{\mu} \phi_{27}^{\alpha} \partial_{\nu} \bar{\phi}_{27}^{\bar{\beta}} G^{\mu \nu} \\
& g_{a \bar{b}}^{(\overline{2 \bar{b}})} \partial_{\mu} \phi_{\frac{a}{27}} \partial_{\nu} \bar{\phi} \frac{\bar{b}}{27} G^{\mu \nu}
\end{aligned}
$$

ii)

$$
\begin{aligned}
& W_{\alpha \beta \gamma}(\varphi) \phi_{27}^{\alpha} \phi_{27}^{\beta} \phi_{27}^{\gamma} \\
& W_{a b c}(\psi) \phi \frac{a}{27} \phi \frac{b}{27} \phi \frac{c}{27}
\end{aligned}
$$

iii)

$$
\frac{1}{g^{2}(S, \varphi, \psi)} T_{r} F_{\mu \nu} F^{\mu \nu}+\theta(S, \varphi, \psi) T_{r} F_{\mu \nu} \tilde{F}^{\mu \nu}
$$

where $M^{i}$ denote any of the two sets of moduli $\varphi^{\alpha}, \psi^{a}, S=D+i a$ is the dilatonaxion scalar field, $g_{i \bar{j}}, g_{\alpha \bar{\beta}}^{(27)}, g_{a \bar{b}}^{(27)}$ are the metrics for moduli, families and antifamilies respectively, $W_{a b c}, W_{\alpha \beta \gamma}$ are the Yukawa couplings, $g^{2}$ and $\theta$ are the gauge coupling and the $\theta$-angle for the gauge field strength $F_{\mu \nu}\left(\tilde{F}_{\mu \nu}\right.$ is the dual of $\left.F_{\mu \nu}\right)$.

We note that the moduli space $\mathcal{M}$ has the structure of a product space

$$
\mathcal{M}=\mathcal{M}_{1} \times \mathcal{M}_{2}
$$

where $\mathcal{M}_{1}, \mathcal{M}_{2}$ are parametrized by the $\varphi^{\alpha}$ and $\psi^{a}$, respectively. This can be proven either in SCFT or by supersymmetry arguments ${ }^{7}$. The two spaces are actually related by the mirror hypothesis ${ }^{8}$ which establishes that exchanging the role of the two parameters $\varphi^{\alpha}, \psi^{a}$ associated to the Kähler class and complex structure deformations of the C.Y. 3-fold,one obtains a mirror C.Y.-3-fold in which the $H^{(2)}$ and $H^{(3)}$ cohomology classes are exchanged, due to the isomorphism of the underlying SCFT's under sign change of the $U(1)$-charge. In the following we shall restrict our attention only to the manifold $\mathcal{M}$ of the complex structure moduli. The results thus obtained are also valid for the moduli space of the Kähler class deformations of the associated mirror C.Y. manifold. 
The structure of the quantities appearing in eq.s (1.1-6) is completely determined by the local and global properties of the moduli space. These properties in turn are encoded in the Picard-Fuchs equations for the periods $X^{A}(\psi), F_{A}(\psi)$ of the Calabi-Yau family parametrized by the $\psi^{a^{\prime}}$ s. Once we know the periods we may reconstruct the Kähler potential of the moduli space and the Yukawa couplings as follows ${ }^{6}$ :

$$
\begin{gathered}
K(\psi, \bar{\psi})=-\log i\left(X^{A} \bar{F}_{A}-\bar{X}^{A} F_{A}\right) \\
W_{a b c}=\frac{\partial X^{A}}{\partial \psi^{a}} \frac{\partial X^{B}}{\partial \psi^{b}} \frac{\partial X^{C}}{\partial \psi^{c}} \frac{\partial^{3} F}{\partial X^{A} \partial X^{B} \partial X^{C}}
\end{gathered}
$$

where $F=F\left(X^{A}\right)$ is a degree two homogeneous function such that

$$
X^{A} F_{A}=2 F \quad \rightarrow \quad F_{A} \equiv \frac{\partial F}{\partial X^{A}}
$$

Notice that the characterization of the local geometry of the moduli space given by eq.s (1.8-1.10) coincides with the definition of Special Kähler ${ }^{9,10,11}$ geometry and indeed the periods $\left(X^{A}, F_{A}\right)$ of the Calabi-Yau 3-fold can be indentified with a set of globally defined holomorphic sections in terms of which the special Kähler geometry can be defined. In this lecture however we will be mainly concerned with the global properties of the moduli space $\mathcal{M}$ which are consequence of the fact that $\mathcal{M}$ possesses a group $\Gamma$ of discrete isometries which is generally referred to as the target space duality group, or modular group ${ }^{12}$. The duality group describes quantum symmetries of the string effective actions and is the discrete version of the non-compact symmetries of old supergravity Lagrangians (no-scale supergravities). The most celebrated example is the effective Lagrangian obtained by compactification of the heterotic string on a 6-torus, modded out by some discrete symmetry ( orbifold ) with residual $N=1$ space-time supersymmetry. The Kähler class untwisted modulus (corresponding to the volume size) $t=2\left(R^{2}+\right.$ $i \sqrt{|g|}$ ) parametrizes the homogeneous space ${ }^{13}$ :

$$
\frac{S U(1,1)}{U(1)}
$$

This sector of the theory is obviously invariant under $\operatorname{PSL}(2, \mathbf{R})$ The Kähler potential of the scalar fields $t, \phi_{27}$ is 


$$
K=-3 \log \left[i(t-\bar{t})-\phi_{27} \bar{\phi} \overline{27}\right]
$$

and the superpotential $\mathcal{W}$ is given by $\mathcal{W}=C \phi_{27}^{3}$ corresponding to a constant Yukawa coupling, $W=C$. The theory is obviously invariant under

$$
t^{\prime}=\frac{a t+b}{c t+d} \quad, \quad \phi_{27}^{\prime}=-\frac{1}{c t+d} \phi_{27} \quad, \quad a d-b c=1
$$

However, when other (twisted) sectors are introduced and/or quantum corrections computed, the duality (quantum symmetry) group is given by $S L(2, \mathbf{Z})$ with generators

$$
t \rightarrow t+1 \quad t \rightarrow \frac{-1}{t}
$$

For Calabi-Yau manifolds $W$ is no longer a constant, but in general it depends on the moduli : $W_{\alpha \beta \gamma}=W_{\alpha \beta \gamma}(M)$ because of the instanton corrections to the $\sigma-$ model perturbative result ( large radius limit). In the one modulus case, however, it turns out that $t \rightarrow t+1$ is still an exact symmetry if $t$ is the special coordinate of the Kähler Special Geometry, or, equivalently, the flat coordinate of the associated topological field theory. Actually one is led to consider the possibility that the translational symmetry is exact for any number of moduli since invariance under $t^{a} \rightarrow t^{a}+1, a=1, \ldots, n$ has its stringy origin in the presence of the antisymmetric axion field $B_{i j}$ in the $\sigma$-model action, and means that the $t^{a}$-variables are periodic. For example the Yukawa coupling in the one modulus case can be written as follows

$$
W(t)=\sum_{n=0}^{\infty} d_{n} e^{2 \pi i n t}
$$

and for many variables we have analogous expansions. In the large radius limit, $t \rightarrow i \infty$, eq. (1.15) gives

$$
W(t)=d_{0}=\text { const }
$$

which implies that $\mathcal{F}(t) \equiv\left|X^{0}\right|^{-2} F\left(X^{A}\right)$ is cubic in $t$. Actually the most general prepotential such that $W(t)$ and the Kähler potential (1.8) are invariant under $t \rightarrow t+1$ is (Cecotti et al. $\left.{ }^{7}\right)$ 


$$
F\left(X^{A}\right)=\left(X^{0}\right)^{2}\left[t^{3}+\lambda(t)+P_{2}(t)\right]
$$

where $P_{2}(t)$ is a polynomial of degree two with purely real coefficients and $\lambda(t)$ a periodic function of $t$. If instead invariance under arbitrary shifts is required, $t \rightarrow t+c, c \in \mathbf{R}$, then $\lambda$ is a constant. Note that a non vanishing $\lambda$ is generated in $\sigma$-model perturbation theory at the four loop level ${ }^{4}$. Under the inversion $t \rightarrow \frac{-1}{t}$ the Kähler potential transforms as follows

$$
K \rightarrow K+f(t)+f(\bar{t})
$$

so that $W$, which has a non trivial Kähler weight undergoes a non trivial transformation.

In the next section we will show that the generalization of the inversion for a generic duality group is given by

$$
t^{a} \rightarrow f^{a}\left(t^{a}, \partial_{a} \mathcal{F}, \mathcal{F} ; A, B, C, D\right) \quad a=1, \ldots, n
$$

where $n$ is the number of moduli, $\mathcal{F}\left(t^{a}\right)=\left(X^{0}\right)^{-2} F\left(X^{A}\right)$ and $A, B, C, D$ are $(n+1) \times(n+1)$ matrices parametrizing a generic $S p(2 n+2 ; \mathbf{Z})$ transformation $M$ :

$$
M=\left(\begin{array}{cc}
A & B \\
C & D
\end{array}\right) \quad \in \quad S p(2 n+2 ; \mathbf{Z})
$$

For example in the case of the quintic studied by Candelas et al. the duality group acting on the single modulus $t$ is generated by two transformations:

$$
\begin{gathered}
t \rightarrow t+1 \\
t \rightarrow \frac{t}{t \mathcal{F}_{t}^{\prime}-2 \mathcal{F}+1}
\end{gathered}
$$

The knowledge of the duality group is very important since the physical quantities appearing in the effective Lagrangian must transform in a definite way under the group: for example the gauge coupling $g_{a}^{-2}(t, \bar{t})$ is a real function which must be modular invariant in $t, \bar{t}$. 
Moreover some of the duality transformations may induce target-space anomalies in the effective Lagrangian ${ }^{14}$. These anomalies arise when the Kähler potential is not invariant under the duality transformations, but undergoes the transformation (1.18). In this case all the Kähler gauge dependent quantities get transformed and anomalies can be generated. From the above discussion we see that in general possibly anomalous transformations belong to the coset $\frac{\Gamma}{\mathcal{T}}$ where $\Gamma$ is the duality group and $\mathcal{T}$ generates the discrete translations under which the Kähler potential is invariant. The modular group described here acts on the special coordinates $t^{a}$ which are the coordinates which flatten the holomorphic connection of the associated topological field theory. The modular coordinate $\gamma$ on which the two transformations described earlier act as $S L(2, R)$ transformations (for any $F$-function) is described in the next section and the relation of this coordinate with the special coordinate as well as with the Landau-Ginzburg coordinate of the defining polynomial in $\mathrm{CP}(d+1)$ will also be given. In this respect the target space duality group $\Gamma$ is closely related to the monodromy group $\Gamma_{M}$ of the PicardFuchs equations which determine the period matrix for Calabi-Yau manifolds, the precise relation being discussed in the following.

\section{Monodromy of the Picard-Fuchs equations and the quantum modular group}

The periods $\left(X^{A}, F_{A}\right)$ of a given family of Calabi-Yau d-folds are known to satisfy a coupled set of linear partial differential equations called Picard-Fuchs equations (PFE). A general property of these equations is that they are of Fuchsian type, that is they only have regular singular points. They can be derived from the defining polynomial equation $W\left(y^{i}, \psi^{\alpha}\right)=0$ of the Calabi-Yau manifold by using simple algorithms which have been described in ref. [15]. If one solves the PFE for the periods and uses eq.s (1.8) and (1.9), then one reconstructs the Kähler potential of the moduli space and the Yukawa couplings associated to that particular class of C.Y. compactifications. Furthermore the PFE also encode essential informations on the global structure of the moduli space through their monodromy group.

Let us denote by $\Gamma$ the target space duality group (quantum modular group) and by $\Gamma_{W}$ the group of invariance of the superpotential $W\left(y^{i}, \psi^{a}\right) . \Gamma_{W}$ consists 
of these diffeomorphisms of the moduli $\psi^{\alpha}$ which leave $W=0$ invariant except for a (quasi)-homogeneous change of the $C P(d+1)$ coordinates:

$$
W\left(y^{i}, \psi^{\alpha}\right)=0 \quad \underset{\Gamma_{W}}{\longrightarrow} W\left(\tilde{y}^{i}(y) ; \tilde{\psi}^{\alpha}(\psi)\right)=0
$$

where $\tilde{y}^{i}=U_{j}^{i} y^{j}$ and $i, j$ run over all chiral fields with same $U(1)$ charge.

Finally let us denote by $\Gamma_{M}$ the monodromy group of the PFE's. To define it in the simplest way we restrict our attention to the case of one single modulus, in which case the PFE's are ordinary differential equations.

Then, if we denote by $\left(f_{1}(z), \ldots, f_{n}(z)\right)$ a basis of solutions of the differential equation at a point $z$, by analytically continuing $\left(f_{1}, \ldots, f_{n}\right)$ along a closed loop around a singularity $z_{1}$ of the equation we arrive at a new solution at $z_{1}$ which must therefore be expressible as a linear combination of the basis $\left(f_{1}, \ldots, f_{n}\right)$ :

$$
\left(f_{1}, \ldots, f_{n}\right) \rightarrow\left(\hat{f}_{1}, \ldots, \hat{f}_{n}\right)=\left(f_{1}, \ldots, f_{n}\right) A_{z_{1}}
$$

where the $n \times n$ non singular matrix $A_{z_{1}}$ defines the monodromy around $z_{1}$. If the equation has $r$ singular points we obtain $r$ monodromy matrices $A_{z_{1}}, \ldots, A_{z_{r}}$, and if we compose closed loops around $z_{i}$ and $z_{j}$ in the usual way it is clearly seen that to the loop $\gamma_{i} \circ \gamma_{j} \equiv \gamma_{i j}$ encircling $z_{i}$ and $z_{j}$ corresponds the monodromy matrix $A_{z_{j}} \cdot A_{z_{i}}$, and that more generally $A_{z_{1}}, \ldots, A_{z_{r}}$ generate a group, the monodromy group of the differential equation (here the inverse $A_{z_{i}}^{-1}$ is the matrix obtained by running around $z_{i}$ in the opposite direction, and $\mathbf{1}$ corresponds to a circuit contractible to a point).

It turns out that in the known cases the monodromy group $\Gamma_{M}$ is a normal (generally infinite) subgroup of $\Gamma$ and that

$$
\Gamma / \Gamma_{M} \simeq \Gamma_{W}
$$

Following the proposal of Lerche et al. ${ }^{15}$ we assume that (2.3) is true in general, possibly also for the case of many moduli. Equation (2.3) suggests that in order to reconstruct $\Gamma$ we can compute the monodromy group of the PFE's and the invariance group of $W=0$, so that $\Gamma \simeq \Gamma_{W} \oslash \Gamma_{M}$. In this section we give two explicit examples of such construction: the 1-dimensional C.Y. manifold described by a cubic polynomials in $\mathrm{CP}(2)$ : 


$$
W=\frac{1}{3}\left(y_{1}^{3}+y_{2}^{3}+y_{3}^{3}\right)-\psi y_{1} y_{2} y_{3}=0
$$

and the 3-dimensional C.Y. manifold describe by a quintic polynomial in $\mathrm{CP}(4)$ :

$$
W=\frac{1}{5}\left(y_{1}^{5}+y_{2}^{5}+y_{3}^{5}+y_{4}^{5}+y_{5}^{5}\right)-\psi y_{1} y_{2} y_{3} y_{4} y_{5}=0
$$

where $y^{i}, i=1, \ldots, d+2$ are homogeneous coordinates in $\mathrm{CP}(\mathrm{d}+1), d$ is the complex dimension equal to 1 or 3 in our case and $\psi$ is a single modulus parametrizing the complex structure deformations of the hypersurface. Note that while in the case of the torus the space of complex structure deformations is one-dimensional, in the case of the quintic $W_{0}=\frac{1}{5}\left(y_{1}^{5}+y_{2}^{5}+y_{3}^{5}+y_{4}^{5}+y_{5}^{5}\right)$ there are 101 indipendent complex structure deformations so that the moduli space is 101-dimensional. The particular 1-dimensional subspace described by the simple deformation (2.5) is such that $1, y_{1} \ldots y_{5},\left(y_{1} \ldots y_{5}\right)^{2},\left(y_{1} \ldots y_{5}\right)^{3}$ close a subring of the chiral ring of all the marginal operators associated to the deformations of the quintic.

Let us begin to study the case of the torus. From (2.4), using known algorithms, (see ref. [15]), one obtains the following PFE's:

$$
\frac{d}{d \psi}\left(\begin{array}{l}
\omega_{0} \\
\omega_{1}
\end{array}\right)=\left(\begin{array}{cc}
0 & 1 \\
\frac{\psi}{1-\psi^{3}} & \frac{3 \psi^{2}}{1-\psi^{3}}
\end{array}\right)\left(\begin{array}{l}
\omega_{0} \\
\omega_{1}
\end{array}\right)
$$

This can be traded for a single 2nd-order differential equation for $\omega_{0}$

$$
\left(\frac{d^{2}}{d \psi^{2}}-\frac{3 \psi^{2}}{1-\psi^{3}} \frac{d}{d \psi}-\frac{\psi}{1-\psi^{3}}\right) \omega_{0}=0
$$

which exhibits four regular singular points at $\psi^{3}=1, \psi=\infty$.

The monodromy group of this equation can be studied as follows. First of all we note that it is sufficient to compute the monodromy matrix $T_{0}$ around $\psi=1$. In fact the effect of a closed loop around $\psi=\alpha$ and $\psi=\alpha^{2}\left(\alpha=e^{2 \pi i / 3}\right)$ can be computed from the monodromy matrix $T_{0}$ around $\psi=1$ by conjugation with $\mathcal{A}$, where $\mathcal{A}$ represents the operation $\psi \rightarrow \alpha \psi$ :

$$
\begin{aligned}
& T_{1}=\mathcal{A}^{1} T_{0} \mathcal{A}^{-1} \\
& T_{2}=\mathcal{A}^{2} T_{0} \mathcal{A}^{-2}
\end{aligned}
$$

Furthermore a closed loop which encloses all the singular points, including $\infty$, is contractible and therefore 


$$
T_{\infty} T_{2} T_{1} T_{0}=1 \quad \rightarrow \quad T_{\infty}=\left(T_{2} T_{1} T_{0}\right)^{-1}
$$

To compute $T_{0}$ it is convenient to perform the substitution $z=\psi^{3}$ in the differential equation (2.7). We obtain

$$
\left\{9 z(1-z) \frac{d^{2}}{d z^{2}}+(6-15 z) \frac{d}{d z}-1\right\} \omega=0
$$

This is a hypergeometric equation of parameters $a=b=1 / 3, c=2 / 3$ and therefore a set of independent solutions around $z \equiv \psi^{3}=0$ is given by

$$
\left\{\begin{array}{l}
U_{1}=\frac{\Gamma^{2}(1 / 3)}{\Gamma(2 / 3)} F\left(1 / 3,1 / 3,2 / 3 ; \psi^{3}\right) \\
U_{2}=\frac{\Gamma^{2}(2 / 3)}{\Gamma(4 / 3)} \psi F\left(2 / 3,2 / 3,4 / 3 ; \psi^{3}\right)
\end{array}\right.
$$

where $F(a, b, c ; z)$ is the hypergeometric functions.

These 2 solutions can be continued around $\psi^{3}=1$ by known formulae ${ }^{16}$ : one finds

$$
\begin{aligned}
& U_{1}=-\log (1-z) F\left(1 / 3,1 / 3,1 ; 1-\psi^{3}\right)+B_{1}\left(1-\psi^{3}\right) \\
& U_{2}=-\log (1-z) F\left(1 / 3,1 / 3,1 ; 1-\psi^{3}\right)+B_{2}\left(1-\psi^{3}\right)
\end{aligned}
$$

where $B_{1}$ and $B_{2}$ are regular series around $\psi^{3}=1$. (The appearance of the logarithmic factor in (2.12) is traceable to the equality of the roots of the indicial equation around $z \equiv \psi^{3}=1$ ). A closed loop around $\psi=1$ gives

$$
\left(\begin{array}{l}
U_{1} \\
U_{2}
\end{array}\right) \rightarrow\left(\begin{array}{l}
U_{1}^{\prime} \\
U_{2}^{\prime}
\end{array}\right)=\left(\begin{array}{l}
U_{1} \\
U_{2}
\end{array}\right)-2 \pi i F\left(\frac{1}{3}, \frac{1}{3}, 1 ; 1-\psi^{3}\right)\left(\begin{array}{l}
1 \\
1
\end{array}\right)
$$

The Kummer relations ${ }^{16}$ among hypergeometric functions allow us to reexpress $F\left(\frac{1}{3}, \frac{1}{3}, 1 ; 1-\psi^{3}\right)$ in terms of the original basis $\left(U_{1}, U_{2}\right)$ around $\psi=0$,

$$
F\left(\frac{1}{3}, \frac{1}{3}, 1 ; 1-z\right)=\frac{\Gamma\left(\frac{1}{3}\right)}{\Gamma^{2}\left(\frac{2}{3}\right)} F\left(\frac{1}{3}, \frac{1}{3}, \frac{2}{3} ; z\right)+\frac{\Gamma\left(-\frac{1}{3}\right)}{\Gamma^{2}\left(\frac{1}{3}\right)} F\left(\frac{2}{3}, \frac{2}{3}, \frac{4}{3} ; z\right)
$$

Therefore, using the relation $\Gamma(z) \Gamma(1-z)=\frac{\pi}{\sin \pi z}$ one obtains

$$
\left(\begin{array}{l}
U_{1}^{\prime} \\
U_{2}^{\prime}
\end{array}\right)=\left(\begin{array}{cc}
1+i \operatorname{tg} \frac{2 \pi}{3} & i \operatorname{tg} \frac{2 \pi}{3} \\
i \operatorname{tg} \frac{2 \pi}{3} & i-i \operatorname{tg} \frac{2 \pi}{3}
\end{array}\right) \quad\left(\begin{array}{l}
U_{1} \\
U_{2}
\end{array}\right)
$$

that is the monodromy matrix around $\psi=1$ is 


$$
T_{0}=\left(\begin{array}{cc}
1-i \sqrt{3} & i \sqrt{3} \\
-i \sqrt{3} & 1+i \sqrt{3}
\end{array}\right)
$$

To find $T_{1}, T_{2}$ we need to represent $\mathcal{A}: \psi \rightarrow \alpha \psi$ on $U_{1}, U_{2}$. From (2.6), (2.7) and (2.11) we see that under $\psi \rightarrow \alpha \psi$ the differential operator is invariant while

$$
\left(\begin{array}{l}
U_{1} \\
U_{2}
\end{array}\right) \rightarrow\left(\begin{array}{ll}
1 & 0 \\
0 & \alpha
\end{array}\right)\left(\begin{array}{l}
U_{1} \\
U_{2}
\end{array}\right)
$$

Since we are interested in the projective representation of the monodromy group we may rescale our basis in such a way that $\operatorname{det} \mathcal{A}=1$ (note that $T_{0}$ already satisfies $\operatorname{det} T_{0}=1$ ). Hence we have

$$
\mathcal{A}=\left(\begin{array}{cc}
\alpha^{-1 / 2} & 0 \\
0 & \alpha^{1 / 2}
\end{array}\right)
$$

and from $(2.8)$

$$
T_{1}=\left(\begin{array}{cc}
1-i \sqrt{3} & \alpha^{-1} i \sqrt{3} \\
-\alpha i \sqrt{3} & 1+i \sqrt{3}
\end{array}\right) \quad T_{2}=\left(\begin{array}{cc}
1-i \sqrt{3} & \alpha^{-2} i \sqrt{3} \\
-\alpha^{2} i \sqrt{3} & 1+i \sqrt{3}
\end{array}\right)
$$

Let us now recall that the modular group is given by the group of transformations on the variable $\psi$ which leaves the theory invariant. The monodromy group $\Gamma_{M}$ of the PFE's must therefore be a subgroup of the modular group. In our case the modular group of the torus is known a priori to be $\Gamma=S L(2 ; \mathbf{Z})$ and therefore it should be possible to perform a change of basis on the periods $U_{i}$ such that the entries of the generators $T_{0}, T_{1}, T_{2}$ are integer numbers. Actually it is known since the last century that $\Gamma_{M}$ is isomorphic to $\Gamma(3)$, where $\Gamma(3)$ is the group of matrices equivalent to the identity modulo 3 . The basis $\left(\mathcal{F}_{1}, \mathcal{F}_{2}\right)$ where $\Gamma_{M} \simeq \Gamma(3)$ is obtained by the following linear transformation ${ }^{17}$

$$
\left(\begin{array}{l}
\mathcal{F}_{1} \\
\mathcal{F}_{2}
\end{array}\right)=\frac{1}{3\left(1+\alpha^{-1 / 2}\right)}\left(\begin{array}{cc}
3 \alpha^{1 / 2} & -3 \\
1+\alpha^{1 / 2} & \alpha^{2}-1
\end{array}\right) \quad\left(\begin{array}{l}
U_{1} \\
U_{2}
\end{array}\right)
$$

The transformed $\Gamma_{M}$ generators $\hat{T}_{i}$ take the following form:

$$
\begin{gathered}
\hat{T}_{0}=\left(\begin{array}{ll}
1 & 3 \\
0 & 1
\end{array}\right) \quad ; \hat{T}_{2}=\left(\begin{array}{cc}
-5 & 12 \\
-3 & 7
\end{array}\right) \quad ; \hat{T}_{1}=\left(\begin{array}{ll}
-2 & 3 \\
-3 & 4
\end{array}\right) \\
\hat{T}_{\infty} \equiv\left(\hat{T}_{2} \hat{T}_{1} \hat{T}_{0}\right)^{-1}=\left(\begin{array}{cc}
1 & 0 \\
-3 & 1
\end{array}\right)
\end{gathered}
$$


The transformation $\mathcal{A}: \psi \rightarrow \alpha \psi$ is obviously an invariance of $W=0$ (and of the differential operator (2.17)) since it can be undone by the coordinate transformation $y^{i} \rightarrow \alpha^{-1 / 3} y^{i}$. Less evident is the invariance under the transformation $\mathcal{B}:$

$$
\mathcal{B}: \psi^{\prime}=-\frac{\psi+2}{1-\psi}
$$

which can be undone by the change of coordinates

$$
\left(\begin{array}{l}
y_{1}^{\prime} \\
y_{2}^{\prime} \\
y_{3}^{\prime}
\end{array}\right)=\frac{i}{\sqrt{3}}\left(\begin{array}{ccc}
1 & 1 & 1 \\
1 & \alpha & \alpha^{2} \\
1 & \alpha^{2} & \alpha
\end{array}\right)\left(\begin{array}{l}
y_{1} \\
y_{2} \\
y_{3}
\end{array}\right)
$$

In the basis $\left(\mathcal{F}_{1}, \mathcal{F}_{2}\right) A$ and $B$ take the form

$$
\hat{A}=\left(\begin{array}{ll}
1 & -3 \\
1 & -2
\end{array}\right) ; \hat{B}=\left(\begin{array}{ll}
2 & 1 \\
1 & 1
\end{array}\right)
$$

We note that the $\Gamma_{W}$ generators $A, B$ satisfy the relation $A^{3}=B^{2}=1$, $(A B)^{3}=1$ which are the defining relations of the tetrahedral group $\Delta$. Indeed $\Gamma(3)$ is a normal subgroup of $\Gamma \equiv S L(2, \mathbf{Z})$, the modular group of the torus , and $\Gamma / \Gamma(3) \equiv \Gamma_{W}$

We have thus verified that the relation $\Gamma / \Gamma_{M} \simeq \Gamma_{W}$ actually holds in the case of the torus.

Let us now consider the case of the quintic (2.5) describing a C.Y. 3-fold in $\mathrm{CP}(4)$. The PFE for the periods is given by the following fourth-order equation ${ }^{4,5,15}$ :

$$
\frac{d^{4} V}{d \psi^{4}}-\frac{10 \psi^{4}}{1-\psi^{5}} \frac{d^{3} V}{d \psi^{3}}-\frac{25 \psi^{3}}{1-\psi^{5}} \frac{d^{2} V}{d \psi^{2}}-\frac{15 \psi^{2}}{1-\psi^{5}} \frac{d V}{d \psi}-\frac{\psi}{1-\psi^{5}} V=0
$$

The four independent solutions of this equation represent the four periods of the uniquely defined $(3,0)$-form $\Omega$ which always exists on a C.Y. space; in our case it can be explicit computed from the defining polynomial (2.5), but we do not need its explicit form in the following. The periods are defined by integrating $\Omega(y, \psi)$ on a basis $\left(\gamma_{A}, \gamma^{B}\right)$ of 3 -cycles of $W$ satisfying

$$
\gamma^{A} \cap \gamma_{B}=-\gamma_{B} \cap \gamma^{A}=\delta_{B}^{A} \quad ; \quad \gamma^{A} \cap \gamma^{B}=\gamma_{A} \cap \gamma_{B}=0
$$


The basis (2.26) is defined only up $S p(4 ; \mathbf{Z})$ transformations, which leave the intersection properties (2.26) invariant. We define

$$
V=\left(X^{0}, X^{1}, F_{0}, F_{1}\right) \equiv\left(X^{A}, F_{A}\right)
$$

where

$$
\int_{\gamma_{A}} \Omega=X^{A}(\psi) ; \int_{\gamma^{A}} \Omega=F_{A}(\psi)
$$

Actually on a C.Y. 3-fold the four periods $V$ are not functionally independent, but satisfy the relation (1.10) where $F$ is a homogeneous function of degree two in $X^{0}, X^{1}$. That has its counterpart in a functional relation satisfied by the coefficients of the differential equation (2.25), namely ${ }^{18,19}$

$$
W_{3}=a_{1}-\frac{d a_{2}}{d \psi}-\frac{1}{2} \frac{d^{2} a_{3}}{d \psi^{2}}=0
$$

where $a_{i}$ is the coefficients of $\frac{d^{i}}{d z^{i}}$ in the differential equation (2.25).

The reason why we have called $W_{3}$ the l.h.s. of eq. (2.29) is that it coincides with the $W_{3}$ generator of the $W_{4}$-algebra associated to a generic 4 -th order linear differential operator (2.25). Actually $W_{3}=0$ is an invariant statement since $W_{3}$ transforms as a covariant tensor of order 3 under $\psi$-reparametrizations

$$
W_{3}^{\prime}\left(\psi^{\prime}\right)=J^{-3} W_{3}(\psi)
$$

where $J$ is the Jacobian of the transformation. The fact the $W_{3}=0$ for the PicardFuchs equation of the quintic was first noted by Lerche et al. [15]. Actually this constraint has been proven to hold quite generally as a consequence of the fact that the moduli space geometry is a special Kähler geometry ${ }^{18,19}$. Indeed one finds that in the one modulus case $W_{3}=0$ is equivalent to the statement that the associated $4 \times 4$ linear system

$$
\left(\frac{d}{d \psi}+A\right) \hat{V}=0
$$

where $\hat{V} \equiv\left(V, V^{\prime}, V^{\prime \prime}, V^{\prime \prime \prime}\right)^{t}$, has a Drinfeld-Sokolov connection $A$ which is gauge equivalent to a $S p(4)$-connection ${ }^{20}$. 
The gauge group acting on (2.31) is the group generated by the strictly lower triangular matrices which leave invariant the top component of $\hat{V}$ and therefore also the differential equation (2.26).* Note that in the gauge where $A$ is valued in the Lie Algebra of $S p(4)$ the matrix of solutions of (2.31) is an element of $S p(4)$

$$
\hat{V}=\left(\begin{array}{cccc}
V_{1} & V_{2} & V_{3} & V_{4} \\
\star & \star & \star & \star \\
\star & \star & \star & \star \\
\star & \star & \star & \star
\end{array}\right) \in S p(4)
$$

In (2.32) the top row is the set of gauge invariant solutions of (2.25). Of course the gauge (2.32) is not completely fixed since arbitrary $S p(4)$ gauge transformations on (2.31) keep the matrix $\hat{V}$ in the symplectic gauge. Another consequence of the relation of the PFE's with Special Geometry is that the coefficient $a_{3}$ of $\frac{d^{3}}{d z^{3}}$ has always the following form ${ }^{18}$ :

$$
a_{3}=-W^{-1} \frac{d W^{-1}}{d z}
$$

where $W(\psi)$ is the Yukawa coupling of the effective $N=1$ theory obtained by compactification on the given C.Y. 3-fold. As an example in the quintic case by comparing (2.33) with (2.25) we find

$$
W(\psi)=\frac{1}{1-\psi^{5}}
$$

The important thing to observe is that many of the properties that we discussed in the one modulus case can be easily extended to the case of $n$ moduli, where the PFE's are partial differential equations. Indeed, as it has been shown in ref.[19] in the $n$ - moduli case the linear system (2.31) can be generalized as follows:

$$
\left(\frac{\partial}{\partial \psi^{\alpha}}+A_{\alpha}(\psi)\right) V(\psi)=0
$$

where $V$ is now a $2 n+2$-dimensional vector and $A_{\alpha}$ is a 1 -form matrix which in general is valued in the Lie Algebra of $G L(2 n+2)$. The set of $2 n+2$ linearly independent vectors of $(2.35)$ is now given by a $(2 n+2) \times(2 n+2)$ matrix of the form

* If one allows for rescaling of the solutions of (2.25) then the gauge group can be extended to the Borel subgroup of $G L(4)$. 


$$
V=\left(\begin{array}{cccc}
X^{0} & X^{a} & F_{a} & -F_{0} \\
\star_{n x 1} & \star_{n x n} & \star_{n x n} & \star_{n x 1} \\
\star_{n x 1} & \star_{n x n} & \star_{n x n} & \star_{n x 1} \\
\star_{1 x n} & \star_{1 x n} & \star_{1 x}
\end{array}\right)
$$

where $X^{A}=\left(X^{0}, X^{a}\right), F_{A}=\left(F_{0}, F_{a}\right), a=1, \ldots, n$ represent the periods of the holomorphic 3-form $\Omega$ defined in (2.28), with the proviso that the index $A$ now takes the values $A=0,1, \ldots, n$. It can be shown that for C.Y. 3-folds the higher order differential equations equivalent to the linear system (2.35) is always a coupled set of partial differential equations of order four.( Eq.(2.25) is in fact an example of this general rule in the case of a single modulus). This result can be shown in general by using the Special Geometry identities, which are equivalent to the Picard-Fuchs equations ${ }^{19}$.Furthemore the same identities also tell us that one can take advantage of the aforementioned gauge invariance of the linear system (2.35) in such a way that the $A_{\alpha^{-}}$connection becomes valued in the Lie Algebra of $S p(2 n+2)$.Specifically if we perform the gauge transformation

$$
\begin{gathered}
V \rightarrow \mathcal{N} V \quad ; \quad \mathcal{N}=\left(\begin{array}{cccc}
\star & 0_{1 \times n} & 0_{1 \times n} & 0 \\
\star_{n \times 1} & \star_{n \times n} & 0_{n \times n} & 0_{n \times 1} \\
\star_{n \times 1} & \star_{n \times n} & \star_{n \times n} & 0_{n \times 1} \\
\star & \star_{1 \times n} & \star_{1 \times n} & \times
\end{array}\right) \\
A^{\prime}=\mathcal{N}^{-1} A \mathcal{N}+\mathcal{N}^{-1} d \mathcal{N}
\end{gathered}
$$

where $\mathcal{N}$ belongs to the Borel subgroup of $G L(2 n+2)$ then it is always possible to choose $\mathcal{N}$ in such a way that the linear system be transformed in the "Special Geometry gauge",namely:

$$
\left(\partial_{\alpha}+A_{\alpha}\right) V=0
$$

where $A_{\alpha}=\boldsymbol{\Gamma}_{\alpha}+\mathbf{C}_{\alpha}$, is given by:

$$
\boldsymbol{\Gamma}_{\alpha}=\left(\begin{array}{cccc}
-\partial_{\alpha} \hat{K} & 0 & 0 & 0 \\
0 & \left(\hat{\Gamma}_{\alpha}-\partial_{\alpha} \hat{K} \mathbf{1}\right)_{\beta}^{\gamma} & 0 & 0 \\
0 & 0 & \left(\partial_{\alpha} \hat{K} \mathbf{1}-\hat{\Gamma}_{\alpha}\right)_{\gamma}^{\beta} & 0 \\
0 & 0 & 0 & \partial_{\alpha} \hat{K}
\end{array}\right)
$$




$$
\mathbf{C}_{\alpha}=\left(\begin{array}{cccc}
0 & \delta_{\alpha}^{\gamma} & 0 & 0 \\
0 & 0 & \left(W_{\alpha}\right)_{\gamma \beta} & 0 \\
0 & 0 & 0 & \delta_{\alpha}^{\beta} \\
0 & 0 & 0 & 0
\end{array}\right)
$$

The hatted holomorphic connections $\partial_{\alpha} \hat{K}(z)$ and $\hat{\Gamma}_{\alpha \beta}^{\gamma}(z)$ are given by

$$
\begin{aligned}
& \partial_{\alpha} \hat{K}=-\partial_{\alpha} \log X^{0}(z) \\
& \hat{\Gamma}_{\alpha \beta}^{\gamma}=e_{a}^{-1}(z) \partial_{\beta} e_{\alpha}^{a}(z)
\end{aligned}
$$

where $e_{\alpha}^{a}=\partial_{\alpha} t^{a}(z), t^{a}(z)=X^{a} / X^{0}$. They obviously vanish in the "special coordinate" frame $t^{a}=X^{a} / X^{0}, X^{0}=1$. Thus we see in particular that the special coordinates $t^{a}$ coincide with the "flat coordinates" of the Landau-Ginzburg formulation of SCFT. Note that in the special coordinate frame the connection $\mathbf{C}_{\alpha} \equiv A_{\alpha}$. The set of $\mathbf{C}_{\alpha}$-matrices is easily seen to satisfy the following abelian,nilpotent subalgebra of $S p(2 n+2)$ :

$$
\begin{gathered}
\mathbf{C}_{\alpha} \mathbf{C}_{\beta} \mathbf{C}_{\gamma} \mathbf{C}_{\delta}=0 \\
{\left[\mathbf{C}_{\alpha}, \mathbf{C}_{\beta}\right]=0}
\end{gathered}
$$

whose importance for the determination of the duality group of a general C.Y.3fold will be discussed later on. Note also that $\mathbf{C}_{\alpha}$ is given in terms of the Yukawa coupling $W_{\alpha \beta \gamma}$ and that $\mathbf{C}_{\alpha} \mathbf{C}_{\beta} \mathbf{C}_{\gamma}=E W_{\alpha \beta \gamma}$ where $E$ is the $(2 n+2) \times(2 n+2)$ matrix with zeros everywhere except a 1 in the right upper corner.

The holomorphic form of the special geometry equations give more insight into the group-theoretical properties of the Picard Fuchs.equations. Indeed the matrix $A$ of the linear system is not the most general one: in the gauge (2.40-41) one easily verifies that actually it is valued in the Lie algebra of $S p(2 n+2) \subset$ $G L(2 n+2)$. Indeed from $(2.40-41)$ one has

$$
A_{\alpha} Q=\left(A_{\alpha} Q\right)^{t}
$$


where $Q$ is the symplectic metric satisfying $Q^{2}=-1, Q^{t}=-Q$

$$
Q=\left(\begin{array}{llll} 
& & -\mathbf{1}_{n} & \\
& \mathbf{1}_{n} & &
\end{array}\right)
$$

This in turn implies that the period matrix $V$ is valued in the $S p(2 n+2)$ group. In particular the top row $V$ of the gauge invariant solutions is defined only up to symplectic transformations $M$

$$
V^{\prime}=V M, \quad M \in S p(2 n+2)
$$

These transformations leave invariant the Kähler potential defined in (1.8) since it can be rewritten as $K=-\log \left(V(-i Q) V^{\dagger}\right)$. For more details on the many moduli case see Ceresole et al. ${ }^{19}$.

Let us now come back to the discussion of a single modulus. Our aim is to discuss the modular group $\Gamma$ of the moduli space of the quintic polynomial (2.5).

Let us first consider the duality group $\Gamma_{W}$ of the defining polynomial equation $W=0$ given by (2.5). It is obvious that $\mathcal{A}: \psi \rightarrow \alpha \psi$, where $\alpha \equiv e^{2 \pi i / 5}$, is a symmetry of $W=0$ since it can be undone by a rescaling of the CP (4) homogeneous coordinates: $\left(y_{1}, y_{2}, y_{3}, y_{4}, y_{5}\right) \rightarrow\left(\alpha^{-1} y_{1}, y_{2}, y_{3}, y_{4}, y_{5}\right)$. Obviously $\mathcal{A}^{5}=1$ and this excludes a priori the possibility that we can represent the modular group as a subgroup of $S L(2 ; \mathbf{Z})$ acting projectively on a function of $\psi$, since $S L(2, \mathbf{Z})$ does not possess elements of order 5 . Since there are apparently no other $\psi$-transformations which can be undone by linear transformations of the $y_{i}^{\prime} \mathrm{s}, \Gamma_{W}$ is simply the cyclic group $\mathbf{Z}_{5}$. According to our previous discussion to reconstruct the full modular group we must now compute the monodromy group $\Gamma_{M}$ of eq. $(2.25)$.

$\Gamma_{M}$ will be represented by $4 \times 4$ matrices on the four periods $\left(V_{1}, V_{2}, V_{3}, V_{4}\right)$ solutions of (2.25). The same is true for $\Gamma_{W}$ since $\mathcal{A}: \psi \rightarrow \alpha \psi$ leaves invariant the differential operator of eq. (2.25) and therefore induces just a linear combinations of the periods. Furthermore by using the gauge (2.32),(or,in particular,the Special Geometry gauge), we may represent $\Gamma_{M}$ and $\Gamma_{W}$ by $S p(4 ; \mathbf{Z})$-matrices.

Let us first compute $\Gamma_{M}$. We sketch briefly the procedure, for further details see ref. [4]. 
The differential equation (2.25) is a Fuchsian equation with regular singular points at $\psi=\alpha^{k},(k=0,1, \ldots, 4), \alpha=e^{2 \pi i / 5}$, and $\psi=\infty$. As in the case of the torus it is sufficient to study the monodromy matrix $T_{0}$ around $\psi=1$, since around $\psi=\alpha^{k}$ the corresponding monodromy matrices $T_{k}, k=1,2,3,4$ are given by

$$
T_{0} \rightarrow T_{k}=A^{k} T_{0} A^{-k}
$$

where $A$ represents $\psi \rightarrow \alpha \psi$. The monodromy around $\psi=\infty$ depends on the other generators around $\psi=\infty$ through the relations $T_{\infty} T_{4} T_{3} T_{2} T_{1} T_{0}=1$.

As in the torus case it is convenient to transform eq. (2.25) into a generalized hypergeometric equation through the substitution $z=\psi^{-5}$. We obtain

$$
\begin{aligned}
\left\{\frac{d^{4}}{d z^{4}}\right. & -\frac{2(4 z-3)}{z(1-z)} \frac{d^{3}}{d z^{3}}-\frac{72 z-35}{5 z^{2}(1-z)} \frac{d^{2}}{d z^{2}}-\frac{24 z-5}{5 z^{3}(1-z)} \frac{d}{d z}- \\
& \left.-\frac{24}{625 z^{3}(1-z)}\right\} V(z)=0
\end{aligned}
$$

which has singular Fuchsian points at $z=0,1, \infty$ with associated Riemann $\mathrm{P}-$ symbol

$$
P\left\{\begin{array}{cccc}
0 & \infty & 1 & \\
0 & 1 / 5 & 0 & \\
0 & 2 / 5 & 1 & ; \psi^{-5} \\
0 & 3 / 5 & 2 & \\
0 & 4 / 5 & 1 &
\end{array}\right\}
$$

We notice that in the variable $z=\psi^{-5}$ we have introduced a new singular point around $\psi=0$ so that the monodromy around $z=\infty$ corresponds exactly to the representation of $\psi \rightarrow \alpha \psi$ on the periods. In other words the duality generator $A$ becomes part of the monodromy generators of the new equation $(2.50){ }^{*} \mathrm{~A}$ solution of $(2.50)$ around $z=0(\psi=\infty)$ is given by

$$
\omega_{0}(\psi)={ }_{4} F_{3}\left(\frac{1}{5}, \frac{2}{5}, \frac{3}{5}, \frac{4}{5} ; 1,1,1 ; \psi^{-5}\right)
$$

* The same observation can be done in the case of the torus previously discussed. 
In order to represent $A$ in a simple way we may construct a basis of solutions around $\psi=0$ as follows ${ }^{4}$. We first continue $\omega_{0}(\psi)$ around $\psi=0$ by using a Barnes-type integral representation and we obtain:

$$
\omega_{0}(\psi)=-\frac{1}{5 \cdot 16 \pi^{4}} \sum_{n=0}^{\infty} \frac{\Gamma^{5}\left(\frac{n}{5}\right)}{\Gamma(n)}\left(\alpha^{n}-1\right)^{4}(5 \psi)^{n} \quad(\mid \psi<1)
$$

Then we recall that $\psi \rightarrow \alpha \psi$ leaves the differential operator (2.25) invariant so that

$$
\omega_{j}(\psi) \doteq \omega_{0}\left(\alpha^{j} \psi\right) \quad j=0,1,2,3,4
$$

are also solutions of (2.50). The five functions $\omega_{j}$ are subject to the linear relations $\sum_{n=0}^{4} \omega_{j}=0$, as it follows from their explicit expression by the power series (2.53) and the analogous ones derived from (2.54).

If we take $\omega_{0}, \omega_{1}, \omega_{2}, \omega_{4}$ as a basis of solutions around $\psi=0$ it follows immediately that $\psi \rightarrow \alpha \psi$ is represented on $\left(\omega_{2}, \omega_{1}, \omega_{0}, \omega_{4}\right)^{t}$ as follows

$$
A=\left(\begin{array}{cccc}
-1 & -1 & -1 & -1 \\
1 & 0 & 0 & 0 \\
0 & 1 & 0 & 0 \\
0 & 0 & 1 & 0
\end{array}\right)
$$

Next one examines the monodromy $T_{0}$ around $\psi=1$. For this purpose one observes that since $z=1$ has the double root $\rho=1$ for the indicial equation, the continuation of the series $\omega_{j}(\psi),|\psi|<1$, to the neighbourhood $|\psi-1|<1$ will contain logarithms. Indeed one can write

$$
\omega_{j}(\psi)=\frac{1}{2 \pi i} c_{j} \tilde{\omega}(\psi) \log (\psi-1)+r e g .
$$

where $\tilde{\omega}$ is a linear combination of regular solutions around $\psi=1$. It turns out that

$$
\left.\tilde{\omega}(\psi)=-\frac{1}{c_{1}}\left(\omega_{1}(\psi)-\omega_{0}(\psi)\right)=\frac{1}{c_{1}}(\psi-1)+O(\psi-1)^{2}\right)
$$

It follows 


$$
\begin{aligned}
\frac{d \omega_{j}}{d \psi} & =\frac{1}{2 \pi i} c_{j}\left(\frac{d \tilde{\omega}}{d \psi} \log (\psi-1)+\tilde{\omega}(\psi) \frac{1}{\psi-1}+\ldots . .\right) \\
& ={ }_{\psi \rightarrow 1} \frac{1}{2 \pi i} \frac{c_{j}}{c_{1}} \log (\psi-1)+\cdots
\end{aligned}
$$

We see that in order to compute the monodromy coefficients $c_{j}$ one has to compute the asymptotic behaviour of $\frac{d \omega_{j}}{d \psi}$ and look at the coefficient of $\log (\psi-1)$. Using the series expansion for $\omega_{j}$ derived from (2.39) and (2.40) one finds (see [4] for details)

$$
c_{j}=(1,1,-4,6,-4)
$$

From eqs. (2.56-59), one easily finds that the monodromy matrix around $\psi=1$ acting on the basis $\left(\omega_{2}, \omega_{1}, \omega_{0}, \omega_{4}\right)^{t}$ is given by

$$
T_{0}=\left(\begin{array}{cccc}
1 & 4 & -4 & 0 \\
0 & 0 & 1 & 0 \\
0 & -1 & 2 & 0 \\
0 & 4 & -4 & 1
\end{array}\right)
$$

The matrices $A$ and $T_{0}$ given by eqs. (2.55) and (2.60) are integer valued, but not symplectic, since the $\omega_{j}$-basis is not a symplectic basis. According to our previous discussion there must exist a matrix $m$ such that

$$
\hat{T}_{0}=m T_{0} m^{-1} \quad ; \quad \hat{A}=m A m^{-1}
$$

are not only integer--valued but also symplectic.

A solution for $m$ has been found in ref. [4] which is unique up to $S p(4 ; \mathbf{Z})$ transformations.

We choose the following solution:

$$
\hat{A}=\left(\begin{array}{cccc}
1 & -1 & -5 & 3 \\
0 & 1 & 8 & 5 \\
1 & -1 & -4 & 3 \\
0 & 0 & 1 & 1
\end{array}\right) \quad ; \quad \hat{T}_{0}=\left(\begin{array}{cccc}
1 & 0 & 0 & 0 \\
0 & 1 & 0 & 0 \\
-1 & 0 & 1 & 0 \\
0 & 0 & 0 & 1
\end{array}\right)
$$

which act on the right of the row vector (2.27). The other monodromy generators $\hat{T}_{k}, \hat{T}_{\infty}$ around $\psi=\alpha^{k}$ and $\psi=\infty$ are finally computed from eqs. (2.49) and $T_{\infty}=\left(T_{4} T_{3} T_{2} T_{1} T_{0}\right)^{-1}$. 
The conclusion is the following: the duality group $\Gamma$ of the moduli space of the C.Y. 3 -fold (2.5) can be given a $4 \times 4$ representation on the integer valued and symplectic basis of the periods. $\Gamma$ is a subgroup of $S p(4 ; \mathbf{Z})$ generated by the matrices $\hat{A}, \hat{T}_{k}(k=0,1,2,3,4)$, where $\hat{A}$ is a representation of the $\mathbf{Z}_{5}$ subgroup of $S p(4 ; \mathbf{Z})$ which leaves $W=0$ invariant and the $\hat{T}_{k}$ 's generate the monodromy group of the PFE (2.25).

We have already observed that the group $\Gamma$ cannot be a subgroup of $S L(2, \mathbf{Z})$ since $S L(2, \mathbf{Z})$ does not contain elements of order 5 . We may however represent $\Gamma$ as a subgroup of $S L(2, \mathbf{R})$ since $\mathbf{Z}_{5} \in S L(2, \mathbf{R})$. To find the representation we need a variable $\gamma(\psi)$ such that $\mathcal{A}: \psi \rightarrow \alpha \psi$ and the transport around $\psi=1$ are represented as $\operatorname{PSL}(2, \mathbf{R})$ transformations on $\gamma(\psi)$. The determination of $\gamma(\psi)$ and the associated $2 \times 2$ representation of $\Gamma \in S L(2, \mathbf{R})$ has been given in ref. [4] by requiring that $\gamma(\psi)$ be a modular parameter on which $A$ and $T_{0}$ act as transformations of order five and infinity, respectively, on the upper $\gamma$-plane. Standard formulae of the theory of the automorphic functions then determine $\gamma(\psi)$. We give here a different, but closely related derivation, which is based only on the structure of the PFE (2.25).

Let us observe that if we denote by $V(\psi(\gamma))$ the four-dimensional row vector of the periods as a function of $\gamma$, then we must have:

$$
V\left(\psi\left(\frac{a_{i} \gamma+b_{i}}{c_{i} \gamma+d_{i}}\right)\right)=V(\psi(\gamma)) \Gamma_{i}
$$

where $\Gamma_{i}$ is any of the matrices $\hat{A}, \hat{T}_{k}$ and $S_{i} \equiv \frac{a_{i} \gamma+b_{i}}{c_{i} \gamma+d_{i}}$ the corresponding 2dimensional action on $\gamma$. If $V$ is required to be a uniform function of $\gamma$, then $\psi=\psi(\gamma)$ must be uniform and such that the entire $\psi^{5}$-plane is mapped into a fundamental region of the $\gamma-$ plane for the group $\Gamma \equiv\left\{S_{i}\right\}$. That amounts to say that $\gamma$ is a modular variable and $\psi$ is an automorphic function of $\gamma$ with respect to $\Gamma$. There is a general procedure to construct the uniformizing variable $\gamma$ directly from the PFE for $V$. It consists in associating to the main differential equation, eq. (2.50) in our case, a second order differential equation with the same singular points ( $z=0,1, \infty$ in our case) and with exponents determined as follows. If all the integrals of the main equation are regular around the given singularity (no two roots of the indicial equation differ by integers) and if all the roots are 
commensurable quantities multiple of $1 / k$ ( $k$ integer), then the difference of the roots of the indicial equations of the associated 2nd-order equation is taken equal to $1 / k$. In all the other cases the difference of roots is taken equal to zero. The uniformizing variable $\gamma$ is then given by the ratio of two solutions of the associated 2nd-order equation. Let us see how this works in our case. To adhere to the same notations as in [4] we perform the substitution $z \rightarrow \frac{1}{z}$ in the equation (2.50). The P-Riemann symbol (2.37) becomes transformed into

$$
P\left(\begin{array}{cccc}
0 & \infty & 1 & \\
1 / 5 & 0 & 0 & \\
2 / 5 & 0 & 1 & ; \psi^{5} \\
3 / 5 & 0 & 2 & \\
4 / 5 & 0 & 1 &
\end{array}\right)
$$

From (2.64) we see that at $z^{-1} \equiv \psi^{5}=0$ all the roots are multiple of $\frac{1}{k} \equiv \frac{1}{5}$ and do not differ by integers. At $z^{-1}=\infty$ and $z^{-1}=1$ instead we have at least two coincident roots. Therefore calling $\lambda, \mu, \nu$ the differences of the roots of the indicial equation for the associated 2 nd-order equation we have

$$
\lambda=1 / 5 \quad ; \quad \mu=\nu=0
$$

Given the exponent we can immediately write down the associated 2ndorder equation, which, having regular singular points at $z=0,1, \infty$ is a hypergeometric equation of parameters

$$
a=\frac{1}{2}(1-\lambda-\mu+\nu)=\frac{2}{5} ; b=\frac{1}{2}(1-\lambda-\mu-\nu)=\frac{2}{5} ; c=1-\lambda=\frac{2}{5}
$$

that is

$$
z(1-z) \mathcal{F}^{\prime \prime}+\left(\frac{4}{5}-\frac{7}{5} z\right) \mathcal{F}^{\prime}-\frac{4}{25} \mathcal{F}=0
$$

The uniformizing variable is then given by

$$
\gamma=\frac{\mathcal{F}_{1}}{\mathcal{F}_{2}}
$$

where 


$$
\begin{aligned}
& \mathcal{F}_{1} \equiv \frac{\Gamma^{2}\left(\frac{2}{5}\right)}{\Gamma\left(\frac{4}{5}\right)} F\left(2 / 5,2 / 5,4 / 5 ; \psi^{5}\right) \\
& \mathcal{F}_{2} \equiv \frac{\Gamma^{2}\left(\frac{3}{5}\right)}{\Gamma\left(\frac{6}{5}\right)} F\left(3 / 5,3 / 5,6 / 5 ; \psi^{5}\right)
\end{aligned}
$$

are two linearly independent solutions of (2.67). From the theory of the automorphic functions we know that $\gamma$ maps the $\psi^{5}$-plane onto a couple of adjacent triangles inside the circle $|\gamma|^{2}=1$ with internal angles $(0,0, \pi / 5)$; they constitute a fundamental region for the projective action of the modular group $\Gamma$, and the inverse function $\psi=\psi(\gamma)$ is automorphic with respect to $\Gamma$.

It is now easy to derive the explicit representation of $A$ and $T_{0}$ as a subgroup of $S L(2, \mathbf{R})$ on $\mathcal{F}_{1}, \mathcal{F}_{2}$ : one has simply to perform the study of the monodromy group of the differential equation (2.53) in exactly the same way as we did it in the case of the torus, eq. (2.10). One obtains in this case, with completely analogous calculations:

$$
A=\left(\begin{array}{cc}
e^{-i \pi / 5} & 0 \\
0 & e^{i \pi / 5}
\end{array}\right) \quad ; \quad T_{0}=\left(\begin{array}{cc}
1-i \operatorname{tg} \frac{2 \pi}{5} & i \operatorname{tg} \frac{2 \pi}{5} \\
-i \operatorname{tg} \frac{2 \pi}{5} & 1+i \operatorname{tg} \frac{2 \pi}{5}
\end{array}\right)
$$

and

$$
T_{k}=A^{k} T_{0} A^{-k} \quad(k=1,2,3,4) ; \quad T_{\infty}=\left(T_{4} T_{3} T_{2} T_{1} T_{0}\right)^{-1} \equiv(A T)^{-5}
$$

Note that the matrices quoted in the Candelas et al. ${ }^{4}$ are related to those given in (2.57) by the change of basis

$$
\left(\begin{array}{l}
Z_{1} \\
Z_{2}
\end{array}\right)=\left(\begin{array}{cc}
i & -i \alpha^{2} \\
1 & -\alpha^{2}
\end{array}\right)\left(\begin{array}{l}
\mathcal{F}_{1} \\
\mathcal{F}_{2}
\end{array}\right)
$$

which map the interior of the circle $|\gamma|^{2}=1$ into the upper half-plane $\operatorname{Im} \gamma>0$.

Till now we have considered the representation of the modular group $\Gamma$ on the periods as $4 \times 4 S p(4 ; \mathbf{Z})$-valued matrices, or as $S L(2 ; \mathbf{R})$-matrices acting projectively on the unformizing variable $\gamma$. There is however another important variable, the special variable $t$, in terms of which we may give an interesting representation of $\Gamma$ which we now discuss. 
To introduce it let us recall that in general, for any number $n$ of moduli, a symplectic transformation on the period vector $V=\left(X^{A}, F_{A}\right) \equiv\left(X^{0}, X^{a}, F_{0}, F_{a}\right)$, $a=1, \ldots, n$, induces a reparametrization on the special coordinates $t^{a} \equiv \frac{X^{a}}{X^{0}}$. Indeed from

$$
\begin{aligned}
\tilde{V} & =V M \\
M & =\left(\begin{array}{ll}
A & C \\
B & D
\end{array}\right) \quad \in S p(2 n+2, \mathbf{R})
\end{aligned}
$$

where $A, B, C, D$ are $(n+1) \times(n+1)$ matrices obeying

$$
\begin{aligned}
& A^{t} B=B^{t} A \\
& C^{t} D=D^{t} C \\
& A^{t} D-B^{t} C=\mathbf{1}
\end{aligned}
$$

we readily obtain ${ }^{19}$

$$
\tilde{t}^{a}=\frac{\tilde{X}^{a}}{\tilde{X}^{0}}=\frac{A_{B}^{a} X^{B}+B^{a B} F_{B}}{A_{B}^{0} X^{B}+B^{0 B} F_{B}}
$$

Recalling that $F_{A}=\frac{\partial F}{\partial X^{A}}$ where $F(X)$ is a homogeneous function of degree two we have

$$
\begin{aligned}
F\left(X^{A}\right) & =\left(X^{0}\right)^{2} \mathcal{F}\left(t^{a}\right) \\
F_{0}\left(X^{A}\right) & \equiv \frac{\partial F}{\partial X^{0}}=X^{0}\left[2 \mathcal{F}\left(t^{a}\right)-t^{a} \partial_{a} \mathcal{F}\left(t^{a}\right)\right] \\
F_{a}\left(X^{A}\right) & \equiv \frac{\partial F}{\partial X^{a}}=X^{0} \partial_{a} \mathcal{F}\left(t^{a}\right)
\end{aligned}
$$

and substituting in (2.76) we find

$$
\tilde{t}^{a}=\frac{A_{b}^{a} t^{b}+A_{0}^{a}+B^{a b} \mathcal{F}_{b}+B^{a 0}\left(2 \mathcal{F}-t^{b} \mathcal{F}_{b}\right)}{A_{b}^{0} t^{b}+A_{0}^{0}+B^{a b} \mathcal{F}_{b}+B^{00}\left(2 \mathcal{F}-t^{b} \mathcal{F}_{b}\right)}
$$

where $\mathcal{F}_{a} \equiv \partial_{a} \mathcal{F}$. If we now restrict the generic $S p(2 n+2, \mathbf{R})$ matrix $M$ to belong to $\Gamma \subset S p(2 n+2, \mathbf{Z})$ we obtain the representation of $\Gamma$ on the variables $t^{a}$. In particular the subgroup of $S p(2 n+2 ; \mathbf{R})$ consisting of matrices of the form

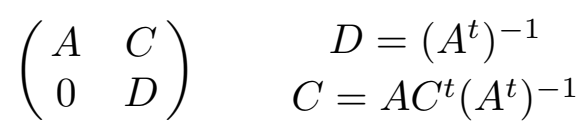


act on the $t^{a^{\prime}} \mathrm{S}$ as a group of linear fractional transformations. It is compelling to assume that the subgroup $\mathcal{T} \subset P S L(2, \mathbf{Z})$ of discrete integer translations

$$
t^{a} \rightarrow t^{a}+n^{a} \quad ; \quad n^{a} \in \mathbf{Z}^{n}
$$

is always contained in $\Gamma$. Indeed the symmetry (2.80) has its stringy origin in the Wess-Zumino term for the $B_{i j}$ axion 2-form in the $\sigma$-model.

Indeed the $\sigma$-model term

$$
\int_{W . S .} d^{2} \sigma \partial_{\alpha} Y^{i} \partial_{\beta} \bar{Y}^{\bar{j}} B_{i \bar{j}} \epsilon^{\alpha \beta}=\int_{T} B_{i \bar{j}} d Y^{i} \wedge d \bar{Y}^{\bar{j}}
$$

where $T$ is the image of the world-sheet (W.S.) in the C.Y. 3-fold, is topological in nature and the $B_{i j}$ are analogous to the $\theta$-parameters of Q.C.D. If the complexified Kähler (1,1)-form is parametrized as

$$
g_{i \bar{j}}+i B_{i \bar{j}}=-i \sum_{a=1}^{n} t^{a} L_{i j}^{a}
$$

where the $L_{i \bar{j}}^{a}$ are a basis of the $(1,1)$-cohomology, a shift $t^{a} \rightarrow t^{a}+n^{a}, n^{a} \in \mathbf{Z}$ induces a topologically non trivial mapping from the world sheet to the C.Y. and corresponds to an instanton correction to the $\sigma$-model perturbative result. Such integral shift is an invariance of the quantum action.

In the one modulus case the existence of the translation symmetry $t \rightarrow t+1$ has been verified in the case of the quintic by Candelas et al. ${ }^{4}$ and proven for a large class of 3 -fold by Morrison ${ }^{5}$. Let us verify it for the quintic. From the explicit form of the two generator $A, T_{0}$ given by eq. (2.48) we find that on the $\left(X^{0}, X^{1}, F_{0}, F_{1}\right)$-basis

$$
\left(T_{0} A\right)^{-1}=\left(\begin{array}{cccc}
1 & 1 & 5 & -8 \\
0 & 1 & -3 & -5 \\
0 & 0 & 1 & 0 \\
0 & 0 & -1 & 1
\end{array}\right)
$$

so that

$$
\tilde{t} \equiv \frac{\tilde{X}^{1}}{\tilde{X}^{0}}=\frac{X^{1}}{X^{0}}+1 \equiv t+1
$$

The $t$-transformations realized by $\mathrm{A}$ and $T_{0}$ are instead non linear: 


$$
\begin{gathered}
A: \tilde{t}=\frac{X^{1}-\left(X^{0}+F^{0}\right)}{X^{0}+F^{0}}=\frac{t-1-\left(2 \mathcal{F}-t \mathcal{F}^{\prime}\right)}{2 \mathcal{F}-t \mathcal{F}^{\prime}} \\
T_{0}: \tilde{t}=\frac{X^{1}}{X^{0}-F_{0}}=\frac{t}{1-2 \mathcal{F}+t \mathcal{F}^{\prime}}
\end{gathered}
$$

We note that while $\left(T_{0} A\right)^{-1}$ corresponds to a circuit around $z=0$, the monodromy around $\psi=\infty$ is represented by $\left(T_{0} A\right)^{-5}$ so that

$$
\left(T_{0} A\right)^{-5}: \quad \tilde{t} \rightarrow t+5
$$

The transformations (2.87) and (2.85) or (2.86) generate the whole modular group on the $t$-modulus.

Coming back to the case of $n$ moduli $t^{a}$, we may also write down the transformation law of the prepotential $F(X)$ in the general case. Indeed recalling the homogeneity relation $2 F(X)=X^{A} F_{A}$ one finds for a generic $S p(4)$-transformations $(2.74)^{21}$ :

$$
\begin{aligned}
& 2 \tilde{F}(\tilde{X})=\left(X^{A}, F_{A}\right)\left(\begin{array}{ll}
A C^{t} & A D^{t} \\
B C^{t} & B D^{t}
\end{array}\right)\left(\begin{array}{c}
X^{A} \\
F_{A}
\end{array}\right) \\
= & 2 F(X)+2 F_{A}\left(B C^{t}\right)_{B}^{A} X^{B}+X^{A}\left(A C^{t}\right)_{A B} X^{B}+F_{A}\left(B D^{t}\right)^{A B} F_{B}
\end{aligned}
$$

where we have used the conditions of symplecticity of the transposed matrix $M^{t}$ in order to reconstruct $F(X)$ on the r.h.s. of (2.88).

If $M \subset \Gamma$, then $\tilde{F}=F$ since a modular transformation is a discrete isometry. In particular for a translation (2.74) gives

$$
F\left(X^{B} A_{B}^{A}\right)=F\left(X^{A}\right)+X^{A}\left(A C^{t}\right)_{A B} X^{B}
$$

since $B=0$ and $A=\left(\begin{array}{cc}1 & n^{a} \\ 0 & \delta_{b}^{a}\end{array}\right)$. In the $t^{a}$-variables eq. (2.89) becomes

$$
\mathcal{F}\left(t^{a}+n^{a}\right)=\mathcal{F}\left(t^{a}\right)+\left(A C^{t}\right)_{a b} t^{a} t^{b}+2\left(A C^{t}\right)_{0 b} t^{b}+\left(A C^{t}\right)_{00}
$$

Thus $F($ or $\mathcal{F})$ is periodic in the $X^{A}$ (or $\left.t^{a}\right)$ up to quadratic additions. In particular the Yukawa coupling $W_{a b c}=\frac{\partial^{3} \mathcal{F}}{\partial t^{a} \partial t^{b} \partial t^{c}}$ is periodic, $W_{a b c}\left(t^{a}+n^{a}\right)=$ $W_{a b c}\left(t^{a}\right)$ and can be expanded in a multiple Fourier series: 


$$
W_{a b c}\left(t^{a}\right)=\sum_{\vec{m} \in \mathbf{Z}^{n}} d_{a b c}(\vec{m}) e^{2 \pi i \vec{m} \cdot \vec{t}}
$$

or, by changing variables $q_{i}=e^{2 \pi i t_{i}}, i=1, \ldots, n$

$$
W_{a b c}\left(q_{i}\right)=\sum_{\vec{m} \in \mathbf{Z}^{n}} d_{a b c}(\vec{m}) \Pi_{i=1}^{n} q_{i}^{m_{i}}
$$

Note that $q_{i}=0$ means $t_{i} \rightarrow i \infty$, that is large radius limit. Therefore the series (2.92) has a constant term correspondig to $\vec{m}=0$ which gives the constant perturbative Yukawa coupling $d_{a b c}(0)$ while the $\vec{m} \neq 0$ terms classify the instanton corrections to the perturbative result.

In the quintic case the number $d_{111}(m)$ have been identified with the number of rational curves of degree $n$ existing in the quintic ${ }^{4}$. The basic observation now is that the $n$ abelian elements of the quantum duality group, related to the discrete Peccei-Quinn symmetry (2.84) are $n$ symplectic $S p(2 n+2)$-matrices entirely determined by the intersection numbers $d_{a b c}(0)$, which are topological classical objects computable in the large "radius" limit, $t^{a} \rightarrow i \infty$. This can be explicitly shown in the one modulus case using the monodromy properties of the PFE around $q_{i}=0$. It has been verified in a two-moduli case by Candelas et al. ${ }^{22}$.

Actually in the one-modulus case the monodromy matrices can be computed from the $A_{\alpha}$-connection of the linear system (2.31). Using the change of variable $\psi \equiv t \rightarrow \frac{1}{2 \pi i} \log q$ the linear system becomes:

$$
\left[q \frac{\partial}{\partial q}+\frac{1}{2 \pi i} A(q)\right] V(q)=0
$$

The monodromy generator $\mathbf{T}$ around $q=0$ is then given by (see Morrison, ref. $[5])$ :

$$
\mathbf{T}=\exp A(q=0)
$$

and it has the property $(\mathbf{T}-\mathbf{1})^{4}=0$ which corresponds to the maximal nilpotency dictated by the order of the differential equation.

If we now recall the structure of the $\mathrm{A}$-connection in special coordinates (see (2.39-2.41), with $\boldsymbol{\Gamma}=0)$ : 


$$
\frac{d}{d t} V=\mathbf{C}(t) V \equiv\left(\begin{array}{cccc}
0 & 1 & 0 & 0 \\
0 & 0 & W_{t t t} & 0 \\
0 & 0 & 0 & 1 \\
0 & 0 & 0 & 0
\end{array}\right) V
$$

we see that for any $W$

$$
(\exp \mathbf{C}-\mathbf{1})^{4}=0
$$

as a consequence of $\mathbf{C}^{\mathbf{4}}=0$ (eq. 2.44).

Therefore the symmetry $\left(T_{0}\right) A^{-1}: t \rightarrow t+1$, is identified,up to a symplectic transformation, with $\exp \mathbf{C}(t=i \infty)$, where $W(i \infty) \equiv d_{111}$.

In the $n$-moduli case the $n$-monodromy generators $\mathbf{T}_{i}=\exp \mathbf{C}_{i}(i \infty)=$ $\mathbf{1}+\mathbf{L}_{i}$ satisfy the following relations:

$$
\begin{aligned}
{\left[\mathbf{L}_{i}, \mathbf{L}_{j}\right] } & =0 \\
\mathbf{L}_{i} \mathbf{L}_{j} \mathbf{L}_{k} & =d_{i j k} E \\
\mathbf{L}_{i} \mathbf{L}_{j} \mathbf{L}_{k} \mathbf{L}_{l} & =0
\end{aligned}
$$

which follow from eqs. $(2.44),(2.45)$ and the related ensuing observation.

\section{Conclusions}

In theses lectures we have described at some length the mathematical origin of target space duality symmetry, its relation to $N=2$ Superconformal field theories, algebraic geometry and effective actions described by Supergravity Lagrangians.

The important discovery of mirror symmetry in the case of Calabi-Yau string compactifications allows one to compute instanton non-perturbative $\sigma-$ model corrections to the effective action purely in terms of algebraic geometrical methods supplemented with the notion of special geometry. When applied to space-time fermions, target space duality, which is a discrete isometry of the moduli space, is seen as a compensating (field dependent) holonomy transformation. Since fermions live in a complex representation of the holonomy group $H \in U(N)$ ( $N$ is the dimension of the Kähler manifold), target space duality transformations induce $\sigma$-model types of anomalies and in particular mixed $U(1)$ gauge anomalies 14. In supersymmetric effective gauge theories derived from strings the anomaly 
cancellation mechanism which takes place originated from a 4D Green-Schwarz mechanism for the 4D antisymmetric tensor $b_{\mu \nu}$ as well as by the existence of local Wess-Zumino terms which involve automorphic functions of the target space modular group. By supersymmetry they induce a moduli dependence on the gauge coupling constant, $\Delta_{a}$, with important effects for the running toward the string unification scale:

$$
\frac{1}{g_{a}^{2}(\mu)}=k_{a} \frac{1}{g_{\text {string }}^{2}}+\frac{b_{a}}{16 \pi^{2}} \log \frac{M_{\text {string }}^{2}}{\mu^{2}}+\Delta_{a}
$$

where $k_{a}$ are the levels of the Kac-Moody algebra for the gauge group factors $G_{a}, g_{\text {string }}^{-2}$ is the v.e.v. of the dilaton field, $M_{\text {string }}^{2}$ is the string mass scale, $M_{\text {string }}^{2}=a \alpha^{\prime-1}$ ( $a$ numerical constant $)$, and $b_{a}$ is the field theoretic 1 -loop $\beta$-fun ction for the gauge group factor $G_{a}$

$$
b_{a}=-3 T\left(G_{a}\right)+\sum_{R} T(R)
$$

( $T\left(G_{a}\right)$ is the Casimir of the adjoint and $T(R)$ are the quadratic Casimirs of the representations $R$ of chiral multiplets).

For a C.Y. manifold with $G=E_{6} \oplus E_{8}$ the $M_{G U T}$-scale occurs then at the value

$$
M_{X}^{2}=M_{\text {string }}^{2}\left(\frac{Y_{8}}{Y_{6}}\right)^{\frac{1}{b_{8}-b_{6}}} \quad\left(\Delta_{a}=\frac{1}{16 \pi^{2}} \log Y_{a}\right)
$$

where $Y_{a}$ are automorphic functions of the target space duality group $\Gamma$. The non-harmonic part of $\Delta_{a}$ can be entirely computed from quantities in special geometry constructed out of the holomorphic period vector $\left(X^{A}, F_{A}\right)$, namely the $\operatorname{norm} X^{A} \bar{F}_{A}-\bar{X}^{A} F_{A}$ and $\operatorname{det}\left(\frac{\partial^{2} F}{\partial X^{A} \partial X^{B}}\right)$.

Threshold effects at the string scale, due to moduli dependence, may play a crucial role for the discussion of supersymmetry breaking when non-perturbative stringy effects, such as gaugino condensation, are suitably incorporated.

\section{Acknowledgements}

We would like to thank P.Candelas,A. Ceresole,P.Fre',W.Lerche,J.Louis and P.Soriani for enlightening discussion. 


\section{References}

1. P. Candelas, G. Horowitz, A. Strominger and A. Witten, Nucl. Phys. B268 (1985) 46. See also M. Green, J. Schwarz and E. Witten, Superstring Theory (Cambridge, Univ. Press, 1987).

2. E. Martinez, Phys. Lett. B217 (1989) 431;

C. Vafa and N.P. Warner, Phys. Lett. B218 (1989) 51;

W. Lerche, C. Vafa and N. Warner, Nucl. Phys. B324 (1989) 427;

D. Gepner, Phys. Lett. B222 (1989) 207;

P. Howe and P. West, Phys. Lett. B223 (1989) 377;

S. Cecotti, L. Girardello and A. Pasquinucci, Nucl. Phys. B328 (1989) 701;

Int. Jou. Mod. Phys. A6 (1991) 2427;

C. Vafa, Int. J. Mod. Phys. A6 (1991) 2829;

K. Intrilligator and C. Vafa, Nucl. Phys. B339 (1990) 95;

C. Vafa, Mod. Phys. Lett. A4 1615 and Mod. Phys. Lett. A4 (1989) 1169;

S. Cecotti, Int. J. Mod. Phys. A6 (1991) 1749 and Nucl. Phys. B355 (1991) 755;

A. Giveon and D.J. Smit, Mod. Phys. Lett. A6 24 (1991) 2211.

3. E. Witten, Commun. Math. Phys. 117 (1988) 353; 118 (1988) 411 and Nucl. Phys. B340 (1990), 281;

T. Eguchi and S.K. Yang, Mod. Phys. Lett. A5 (1900) 1693;

C. Vafa, Mod. Phys. Lett. A6 (1991) 337;

K. Li, Nucl.Phys. B354 (1991) 711;

B. Blok and A. Varchenko, Int.Jou. Mod. Phys. A7 (1992) 1467;

R. Dijkgraaf, E. Verlinde and H. Verlinde, Nucl. Phys. B348 (1991) 435 and B352 (1991) 59;

A. Giveon and D.J. Smit, Progr. Theor. Phys. Suppl. 102 (1990) 351;

Mod. Phys. Lett. A6 (1991) 2211; Int.Jou.Mod. Phys. A7 (1992) 973;

S. Cecotti and C. Vafa, Nucl. Phys. B367 (1991) 359.

4. P. Candelas, X.C. de la Ossa, P.S. Green and L. Parkes, Phys. Lett. 258B (1991) 118;

P. Candelas, X.C. de la Ossa, P.S. Green and L. Parkes, Nucl. Phys. B359 (1991) 21. 
5. D. Morrison, Essays on Mirror manifolds,S.T.Yau Editor, Intenational Press (1992);

A. Font, Periods and Duality Symmetries in Calabi-Yau Compactifications (preprint UCVFC/DF-1-92);

A. Klemm and S. Theisen, Considerations of one modulus Calabi-Yau compactifications: Picard-Fuchs equations, Kähler potentials and mirror maps (Karlsruhe preprint KA-THEP-03-92).

6. P. Candelas and X.C. de la Ossa, Nucl. Phys. B355 (1991) 455.

7. S. Cecotti, S. Ferrara and L. Girardello, Int.Jou. Mod. Phys. A4 (1989) 2475

S. Cecotti, S. Ferrara and L. Girardello, Phys. Lett. B213 (1988) 443;

L.J. Dixon, V.S. Kaplunovsky and J. Louis, Nucl. Phys. B329 (1990) 27.

8. P.Aspinwall and D. Morrison, Topological field theory and rational curves, preprint DUK-M-91-12;

E. Witten, Essays on Mirror manifolds , S.T.Yau Editor,International Press (1992)

B.R. Greene and M.R. Plesser, Nucl. Phys. B338 (1990) 15;

P. Candelas, M. Linker and R. Schimmrigk, Nucl. Phys. B341 (1990) 383;

P. Aspinwall, C.A. Lütken and G.G. Ross, Phys. Lett. B241 (1990) 373;

P. Aspinwall, C.A. Lütken, Nucl. Phys. B353 (1991) 427 and B355 (1991)

482; See also contributions in "Essay on Mirror Manifolds" edited by S.T.

Yau, International Press (1992).

9. B. de Wit and A. Van Proeyen, Nucl. Phys. B245 (1984) 89

B. de Wit, P. Lauwers and A. Van Proeyen, Nucl. Phys. B255 (1985) 569;

E. Cremmer, C. Kounnas, A. Van Proeyen, J.P. Deredinger, S. Ferrara, B. de Wit and L. Girardello, Nucl. Phys. B250 (1985) 385.

10. A. Strominger, Commun. Math. Phys. 133 (1990) 163.

11. L. Castellani, R. D'Auria and S. Ferrara, Phys. Lett. B241 (1990) 57;

L. Castellani, R. D'Auria and S. Ferrara, Class. Quant. Grav. 1 (1990) 317

R. D'Auria, S. Ferrara and P. Frè, Nucl. Phys. B359 (1991) 705.

12. K. Kikkawa and M. Yamasaki, Phys. Lett. 149B (1984) 357;

N. Sakai and L. Senda, Progr. Theor. Phys. 75 (1986) 692; 
V.P. Nair, A. Shapere, A. Strominger and F. Wilczek, Nucl. Phys. B287 (1987) 402;

A. Giveon, E. Rabinovici and G. Veneziano, Nucl. Phys. B322 (1989) 167;

A. Shapere and F. Wilczek, Nucl. Phys. B320 (1989) 167;

M. Dine, P. Huet and N. Seiberg, Nucl. Phys. B322 (1989) 301;

J. Molera and B. Ovrut, Phys. Rev. D40 (1989) 1150;

J. Lauer, J. Maas and H.P. Nilles, Phys. Lett. B226 (1989) 251 and Nucl. Phys. B351 (1991) 353;

W. Lerche, D. Lüst and N.P. Warner, Phys. Lett. B231 (1989) 418;

M. Duff, Nucl. Phys. B335 (1990) 610;

A. Giveon and M. Porrati, Phys. Lett. B246 (1990) 54 and Nucl. Phys. B355 (1991) 422;

Giveon, N. Malkin and E. Rabinovici, Phys. Lett. B238 (1990) 57;

J. Erler, D. Jungnickel and H.P. Nilles, MPI-Ph/91-90;

S. Ferrara, D. Lüst, A. Shapere and S. Theisen, Phys. Lett. B233 (1989) 147

J. Schwarz, Caltech preprints CALT-6S-1581 (1990) CALT-68-1728 (1991) and CALT-68-1740 (1991);

J. Erler, D. Jungnickel and H.P. Nilles, MPI-Ph/91-81.

13. E. Witten, Phys. Lett. B155 (1985) 151.

14. L.J. Dixon, V.S. Kaplunovsky and J. Louis, Nucl. Phys. B355 (1991) 649; J.P. Deredinger, S. Ferrara, C. Kounnas and F. Zwirner, Nucl. Phys. B372 (1992) 145;

J. Louis, PASCOS, (1991), Proceedings, P. Nath Editor, World Scientific 1991 ;

G.L. Cardoso and B. Ovrut, Nucl. Phys. B369 (1992) 351.

15. A. Cadavid and S. Ferrara, Phys. Lett. B267 (1991) 193;

W. Lerche, D. Smit and N. Warner, Nucl. Phys. B372 (1992) 87;

16. A. Erdélyi, F. Oberhettinger, W. Magnus and F.G. Tricomi, Higher Trascendental Functions Mac Graw-Hill, New York, 1953.

17. P. Frè and P. Soriani, private communication, see also P. Soriani, SISSA Ph.D-thesis.

18. S. Ferrara and J. Louis, Phys. Lett. B278 (1992) 240. 
19. A. Ceresole, R. D'Auria, S. Ferrara, W. Lerche and J. Louis, Int.Jou.Mod.Phys. A8 (1993) 79;

20. P. Di Francesco, C. Itzynkson and J.B. Zuber, Commun. Math. Phys. 140 (1991) 543.

21. M. Villasante, Phys. Rev. D45 (1992) 1831.

22. P.Candelas, private communication. 